Article

\title{
A Multi-Point Method Considering the Maximum Power Point Tracking Dynamic Process for Aerodynamic Optimization of Variable-Speed Wind Turbine Blades
}

\author{
Zhiqiang Yang ${ }^{1}$, Minghui Yin ${ }^{1, *}$, Yan $\mathrm{Xu}^{2}$, Zhengyang Zhang ${ }^{1}$, Yun Zou ${ }^{1}$ and Zhao Yang Dong ${ }^{2}$ \\ 1 School of Automation, Nanjing University of Science and Technology, Nanjing 210094, China; \\ yang-zhiqiang@foxmail.com (Z.Y.); njustautozzy@163.com (Z.Z.); zouyun@vip.163.com (Y.Z.) \\ 2 School of Electrical and Information Engineering, University of Sydney, Sydney, NSW 2006, Australia; \\ yan.xu@sydney.edu.au (Y.X.); zydong@ieee.org (Z.Y.D.) \\ * Correspondence: ymhui@vip.163.com; Tel.: +86-151-0516-8909
}

Academic Editor: Frede Blaabjerg

Received: 15 February 2016; Accepted: 23 May 2016; Published: 31 May 2016

\begin{abstract}
Due to the dynamic process of maximum power point tracking (MPPT) caused by turbulence and large rotor inertia, variable-speed wind turbines (VSWTs) cannot maintain the optimal tip speed ratio (TSR) from cut-in wind speed up to the rated speed. Therefore, in order to increase the total captured wind energy, the existing aerodynamic design for VSWT blades, which only focuses on performance improvement at a single TSR, needs to be improved to a multi-point design. In this paper, based on a closed-loop system of VSWTs, including turbulent wind, rotor, drive train and MPPT controller, the distribution of operational TSR and its description based on inflow wind energy are investigated. Moreover, a multi-point method considering the MPPT dynamic process for the aerodynamic optimization of VSWT blades is proposed. In the proposed method, the distribution of operational TSR is obtained through a dynamic simulation of the closed-loop system under a specific turbulent wind, and accordingly the multiple design TSRs and the corresponding weighting coefficients in the objective function are determined. Finally, using the blade of a National Renewable Energy Laboratory (NREL) 1.5 MW wind turbine as the baseline, the proposed method is compared with the conventional single-point optimization method using the commercial software Bladed. Simulation results verify the effectiveness of the proposed method.
\end{abstract}

Keywords: aerodynamic optimization; closed-loop system; multi-point method; maximum power point tracking (MPPT) control; variable-speed wind turbine (VSWT)

\section{Introduction}

Vigorous development and utilization of wind energy is of great significance to improving energy structure and achieving sustainable development. At present, the employment of horizontal-axis wind turbines (HAWTs) for power generation is the most valuable and widely used form of wind energy utilization. As blade is the key component of wind turbines for wind energy capture, it is critical to investigate its aerodynamic optimization technology in order to improve the efficiency of wind turbines $[1,2]$.

In the conventional methods for aerodynamic optimization of wind turbine blades, the commonly used objective function is to maximize the power coefficient $C_{\mathrm{p}}$ at a particular design tip speed ratio (TSR), i.e., the optimum TSR $\lambda_{\text {opt }}$, such as in the Glauert method [3], Wilson method [4] and other analytical methods [5-7], based on the blade element momentum (BEM) theory with consideration of 
different correction factors. In general, these methods all concentrate on improving the performance at a single operating point.

Because the fixed-speed wind turbine (FSWT) keeps operating at a fixed rotor speed, its operational TSR $\lambda_{\text {ope }}$ varies with wind speed. Therefore, the single-point design methods cannot lead to the optimum design for FSWT blades, since only one TSR in the total operational range is considered [8-13]. In order to take the off-design performance into account, maximum annual energy production (AEP) is regarded as the design goal to increase the total captured wind energy for FSWTs under a specific wind speed frequency distribution [11-13]. References [12,13] have defined the maximization of $C_{p}$ at a single TSR and AEP as the objective function to design FSWT blades, respectively. The simulation results showed that the AEP-optimized blade achieved more AEP due to its flatter $C_{\mathrm{p}}-\lambda$ curve with a larger average $C_{\mathrm{p}}$ in a wider range of $\lambda_{\text {ope }}$.

For a variable-speed wind turbine (VSWT), which has become the mainstream of large-scale wind power generation systems, the off-design performance also needs to be considered in the blade design. Although the maximum power point tracking (MPPT) control strategy is used for VSWTs to maintain $\lambda_{\text {opt }}$ and achieve the maximum power coefficient $C_{\mathrm{p} \text {,max }}$ from cut-in wind speed up to rated speed [14,15], some studies [16-19] have pointed out that VSWTs with large rotor inertia usually track $\lambda_{\text {opt }}$ rather than stay at $\lambda_{\text {opt }}$ because they cannot respond to turbulence instantly, which results in a distribution of $\lambda_{\text {ope }}$. Moreover, if the top of $C_{\mathrm{p}}-\lambda$ curve is sharp, the tracking loss caused by the distribution of $\lambda_{\text {ope }}$ will be deteriorated [16,17].

However, the objective functions of the aerodynamic design for VSWTs are usually defined as the maximization of $C_{\mathrm{p} \text {, max }}$ at $\lambda_{\text {opt }}$ [6,7] or as the AEP only related to $C_{\mathrm{p}, \max }$ [20-22]. Based on the implicit assumption that VSWTs can be maintained at $\lambda_{\text {opt }}$ by the MPPT control, maximizing AEP is equivalent to optimizing the $C_{\mathrm{p} \text {,max }}$ at $\lambda_{\mathrm{opt}}$, which cannot improve the off-design performance for VSWT blades [20-22]. Because the $\lambda_{\text {ope }}$ of VSWTs is determined by the dynamic tracking process of the turbine under turbulence, it is difficult to obtain its distribution law and define the objective function considering the off-design TSR only based on wind speed probability distribution. Then, in [23], the dynamic simulation is used to calculate the AEP, which actually considers the off-design performance for VSWT blades via numerical computation. Furthermore, in this paper the motivation for introducing the off-design performance, the mechanism of multi-point design and its contribution to improving wind energy extraction are studied in order to propose a multi-point aerodynamic optimization method.

Considering the distribution of $\lambda_{\text {ope }}$, the wind energy capture of VSWTs can be effectively improved by focusing on increasing the power coefficient $C_{\mathrm{p}}$ at the important operational TSRs. In this paper, based on a closed-loop model of VSWTs, the distribution of $\lambda_{\text {ope }}$ is interpreted by analyzing the MPPT dynamic process and quantitatively described by the inflow wind energy at $\lambda_{\text {ope }}$ intervals. Furthermore, by constructing an objective function including the important TSRs where the inflow wind energy is primarily distributed, a multi-point aerodynamic optimization method is proposed to maximize the weighted sum of $C_{\mathrm{p}}$ at multiple TSRs. Essentially, the proposed method aims to coordinate the $C_{\mathrm{p}}-\lambda$ curve with the distribution of $\lambda_{\text {ope }}$. Finally, the blade of a National Renewable Energy Laboratory (NREL) 1.5 MW wind turbine is optimized by the proposed method and compared against the conventional single-point method. A dynamic simulation conducted using the Bladed software validates the effectiveness of the proposed method.

\section{Interpretation for the Distribution of Operational Tip Speed Ratio}

In this section, the distribution of operational TSR $\lambda_{\text {ope }}$ is interpreted by analyzing the dynamic process of MPPT based on a closed-loop model of VSWTs. This indicates that the off-design TSR resulted from MPPT dynamic process should be taken into account in the aerodynamic design of VSWT blades. 


\subsection{A Closed-Loop Model of Variable-Speed Wind Turbines}

The diagram of a closed-loop model of VSWTs is shown in Figure 1, which consists of turbulent wind, rotor, drive train, generator and MPPT controller. Note that because the electromagnetic response time is much shorter than the mechanical response, the converter can be ignored and the generator is assumed to instantaneously follow the torque reference issued by the MPPT controller [18] in the closed-loop model.

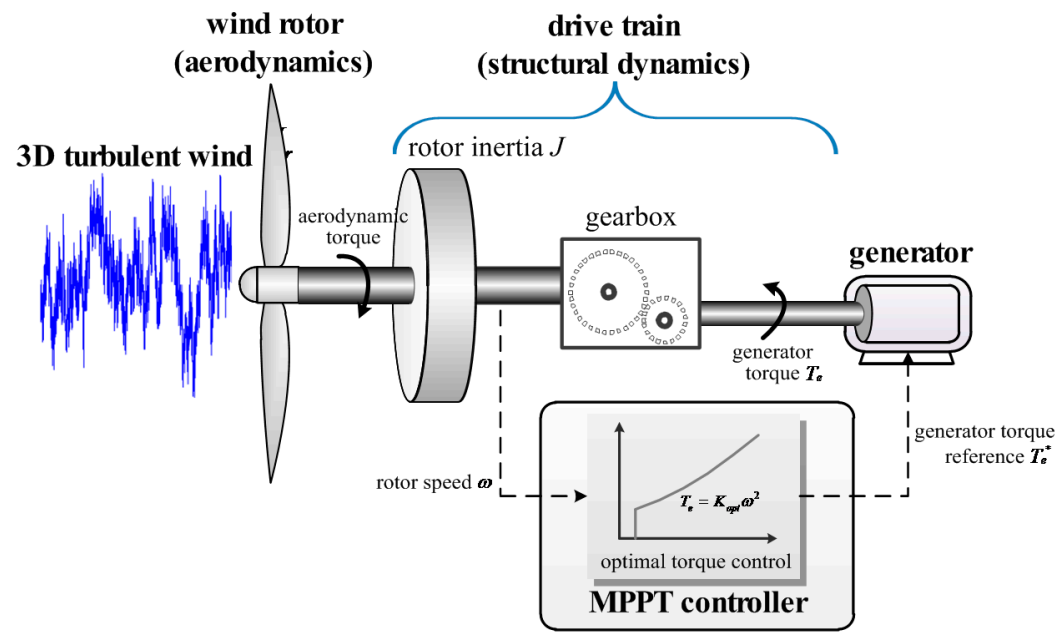

Figure 1. A closed-loop model of variable-speed wind turbines (VSWTs). MPPT: maximum power point tracking.

The commercial software Bladed $[24,25]$ is used in this paper to perform the time domain dynamic simulation of the closed-loop model. This software has passed the Germanischer Lloyd's certification and has been widely applied for wind turbine design and analysis.

The aerodynamic model used within Bladed is based on the classical BEM theory with the consideration of blade tip and hub loss. In order to sufficiently reproduce the dynamics associated with the process, i.e., the vorticity trailed into the rotor wake being affected by the changes in blade loading and the time for the changing of the induced flow field being finite, the dynamic inflow model was selected. This treatment provides a simple, computationally inexpensive and reasonably reliable method of modelling the dynamics of the rotor wake and induced velocity flow field [24].

The commonly-used optimal torque (OT) control $[15,16]$ is employed for MPPT. The principle of this MPPT control is to adjust generator torque according to the rotor speed and a predefined OT versus rotor speed curve that is expressed as:

$$
T_{\mathrm{e}}=K_{\mathrm{opt}} \omega^{2}
$$

where $\omega$ is the rotor speed and:

$$
K_{\mathrm{opt}}=0.5 \rho \pi R^{5} C_{\mathrm{p}, \max } / \lambda_{\mathrm{opt}}^{3}
$$

\subsection{Interpretation for the Distribution of $\lambda_{\mathrm{ope}}$}

The 1.5 MW wind partnership for advanced component technologies (WindPACT) turbine [26] is selected as the reference model. This turbine is developed by NREL and its blade radius $R$ is $35 \mathrm{~m}$, rotor inertia $J$ is $4,740,703 \mathrm{~kg} \cdot \mathrm{m}^{2}$ and optimal TSR $\lambda_{\text {opt }}$ is 6.8 .

To interpret the distribution of $\lambda_{\text {ope }}$ due to the MPPT dynamic process, the responses of wind turbines with different $J$ are compared. By proportionally scaling the mass of each blade element, the rotor inertia of the turbine and its dynamic property can be adjusted accordingly. Note that the 
other parameters including the $C_{\mathrm{p}}-\lambda$ curve, $\lambda_{\mathrm{opt}}, R$ and etc. remain unchanged. Figure 2 shows the trajectories of rotor speed $\omega$ and $\lambda_{\text {ope }}$ under the same step wind speed for the turbines with three different $J$.

Figure 2 shows that:

(1) $\lambda_{\text {ope }}$ equals $\lambda_{\text {opt }}$ only when the rotor speed is regulated at the optimal value by the MPPT control.

(2) Due to the rotor inertia, the turbines cannot instantly respond to sudden changes in wind speed. Consequently, the rotor speed deviates from the optimal value and $\lambda_{\text {ope }}$ deviates from $\lambda_{\text {opt }}$ until the rotor speed is controlled to the optimal value.

(3) With the increase of rotor inertia, the dynamic process of MPPT as well as the duration of $\lambda_{\text {ope }}$ deviating from $\lambda_{\text {opt }}$ is extended, and correspondingly the distribution of $\lambda_{\text {ope }}$ becomes more dispersive.

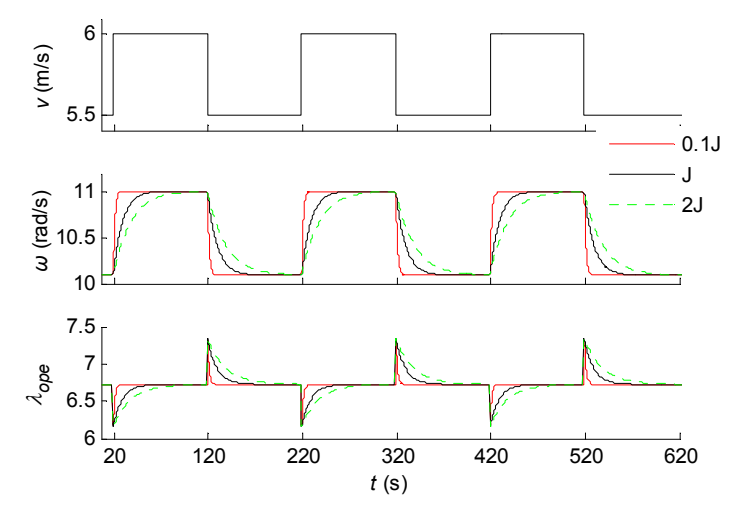

Figure 2. Response of wind turbines with different rotor inertia excited by a same step wind speed.

To sum up, because of the MPPT dynamic process, the VSWT cannot keep operating at the optimal rotor speed, which results in the distribution of the operational TSR. Moreover, the longer the dynamic process lasts, the more time the turbine will take to operate at a TSR which deviates from $\lambda_{\text {opt }}$.

\section{Description for the Distribution of $\lambda_{\text {ope }}$ Based on Inflow Wind Energy}

Since the VSWT cannot maintain $\lambda_{\text {opt }}$ because of the MPPT dynamic process, the off-design performance of the turbine at multiple TSR needs to be considered in the blade design. Furthermore, the basis of multi-point design is the quantitative description for the distribution of $\lambda_{\text {ope }}$ so as to determine the multiple TSR that should be focused on. In this section, from the perspective of a closed-loop system, a method is proposed using the inflow wind energy corresponding to each $\lambda_{\text {ope }}$ interval to quantitatively describe the importance of each operating point.

The wind speed $v_{0, i}$ and rotor speed $\omega_{i}$ at the $i$-th step can be obtained by the time domain dynamic simulation on the closed-loop model of VSWTs. The operational TSR at the $i$-th step $\lambda_{\text {ope }, i}$ can therefore be determined as:

$$
\lambda_{\text {ope }, i}=\omega_{i} R / \nu_{0, i}
$$

The corresponding inflow wind energy in front of the rotor plane is calculated as:

$$
E_{\text {inflow }}^{i}=0.5 \rho \pi R^{2} v_{0, i}^{3} \Delta t
$$

where $R$ is the blade radius, $\rho$ is the air density, and $\Delta t$ is the simulation step.

The distribution range of $\lambda_{\text {ope }}$ can be divided into several continuous intervals:

$$
U_{\lambda_{j}}=\left(\lambda_{j}-\Delta \lambda / 2, \lambda_{j}+\Delta \lambda / 2\right), \quad j=1, \cdots, m
$$


where $m$ is the number of TSR intervals, $\Delta \lambda$ is the length of the interval. The mid-point, lower bound and upper bound of the TSR interval are $\lambda_{j}, \lambda_{j}-\Delta \lambda / 2$ and $\lambda_{j}+\Delta \lambda / 2$, respectively.

The inflow wind energy corresponding to the $\lambda_{\text {ope }, i}$ located in the interval $U_{\lambda_{j}}$ is accumulated as follows:

$$
E_{\text {inflow }}^{U_{\lambda_{j}}}=\sum_{\lambda_{\text {ope }, i} \in U_{\lambda_{j}}} E_{\text {inflow }}^{i}
$$

and Equation (6) means that when the turbine operates at the TSR interval $U_{\lambda_{j}}$, there is an amount of $U_{\lambda_{j}}$
$E_{\text {inflow }}$ inflow wind energy passes the rotor plane.

The proportion of $E_{\text {inflow }}^{U_{\lambda_{j}}}$ to the total inflow wind energy $E_{\text {inflow }}^{\text {total }}$ can be expressed by:

$$
p\left(U_{\lambda_{j}}\right)=E_{\text {inflow }}^{U_{\lambda_{j}}} / E_{\text {inflow }}^{\text {total }}=\sum_{\lambda_{\text {ope }, i} \in U_{\lambda_{j}}} E_{\text {inflow }}^{i} / \sum_{i=1}^{N} E_{\text {inflow }}^{i}=\sum_{\lambda_{\text {ope }, i} \in U_{\lambda_{j}}} v_{0, i}^{3} / \sum_{i=1}^{N} v_{0, i}^{3}
$$

where $N$ is the total number of simulation steps.

The total amount of captured wind energy can be defined as the sum of the captured wind energy when the turbine operates at all $U_{\lambda_{j}}$, i.e.,

$$
E_{\text {cap }}^{\text {total }}=\sum_{j=1}^{m} E_{\text {cap }}^{U_{\lambda_{j}}}
$$

Approximating the power coefficients at the TSR interval $U_{\lambda_{j}}$ as the power coefficient at $\lambda_{j}$, Equation (8) can be rewritten as:

$$
E_{\text {cap }}^{\text {total }}=\sum_{j=1}^{m} E_{\text {cap }}^{U_{\lambda_{j}}} \approx \sum_{j=1}^{m} E_{\text {inflow }}^{U_{\lambda_{j}}} C_{\mathrm{p}}^{\lambda_{j}}
$$

As revealed in Equation (9), if the amount of inflow wind energy is larger when the turbine operates at $U_{\lambda_{j}}$, increasing the power coefficient at $\lambda_{j}$ can significantly improve the total amount of captured wind energy. Moreover, $p\left(U_{\lambda_{j}}\right)$ obtained by the statistical results of the dynamic simulation can be regarded as the quantitative index for describing the importance of the increment of $C_{\mathrm{p}}^{\lambda_{j}}$. In the proposed multi-point optimization method (discussed in the following section), this index provides a foundation for determining the objective function, which includes the multiple design TSRs and the corresponding weighting coefficients.

\section{Definition of the Objective Function in the Multi-Point Optimization Problem}

In order to effectively increase the total amount of captured wind energy under turbulent wind, the TSR interval $U_{\lambda_{j}}$ corresponding to larger inflow wind energy is derived based on the index $p\left(U_{\lambda_{j}}\right)$ proposed in Section 3 , and the power coefficient $C_{\mathrm{p}}^{\lambda_{\mathrm{j}}}$ at $\lambda_{j}$ is selectively improved.

The objective function is defined as a sum of the weighted power coefficients at several TSRs:

$$
o b j=\max \sum_{j=1}^{K} \mu_{j} C_{\mathrm{P}}^{\lambda_{j}}
$$

where $K$ is the number of design TSRs, $\mu_{j}$ is the weighting coefficient for $C_{\mathrm{p}}^{\lambda_{j}}$, and subject to $0<\mu_{j}<1$ and $\sum_{j=1}^{K} \mu_{j}=1$.

Furthermore, the detailed steps for determining $\lambda_{j}$ and $\mu_{j}$ are as follows: 
Step 1 Choose the baseline wind turbine (including rotor, drive train and controller) and model it in the Bladed software. Generate the turbulent wind for dynamic simulation according to the annual average wind speed and turbulence class of the wind farm. Determine the simulation parameters, including the simulation time and the step size.

Step 2 Obtain the trajectory of $\lambda_{\text {ope }}$ by the dynamic simulation of the closed-loop model and divide it into several $U_{\lambda_{j}}$ with the interval length $\Delta \lambda$. Then, calculate $p\left(U_{\lambda_{j}}\right)$ according to Equation (7).

Step 3 Choose $U_{\lambda_{j}}$ in a descending order of $p\left(U_{\lambda_{j}}\right)$ until the following condition is satisfied:

$$
\sum_{j=1}^{K} p\left(U_{\lambda_{j}}\right) \geqslant r_{\text {tot }}
$$

Step 4 Regard the mid-points of the multiple $U_{\lambda_{j}}$ chosen in Step 3 as the design TSRs. The weighting coefficients are determined by Equation (12):

$$
\mu_{j}=p\left(U_{\lambda_{j}}\right) / \sum_{j=1}^{K} p\left(U_{\lambda_{j}}\right)
$$

It is noted that $\lambda_{j}$ and $\mu_{j}$ are determined jointly by $\Delta \lambda$ and $r_{\text {tot }}$, which are respectively discussed as follows:

(1) $\Delta \lambda$ is set to $0.25-0.5$. If $\Delta \lambda$ is set to a large value, the curve of $p\left(U_{\lambda_{j}}\right)$ is too rough to describe the distribution of $\lambda_{\text {ope }}$. Moreover, because the $C_{\mathrm{p}}-\lambda$ curve commonly exhibits a parabolic shape, it is hard to precisely modify the $C_{\mathrm{p}}-\lambda$ curve. Therefore, it is unnecessary to calculate $p\left(U_{\lambda_{j}}\right)$ with a very small $\Delta \lambda$.

(2) $r_{\text {tot }}$ is suggested as $90.0 \%$ and thus the union of the chosen TSR intervals can always cover the operational TSR range with the major proportion of inflow wind energy. Actually, it is difficult and unnecessary to increase the power coefficients at all operational TSRs, because improving the power coefficients at the TSR intervals with very small $p\left(U_{\lambda_{j}}\right)$ cannot effectively contribute to the improvement of wind energy capture.

It can be observed from the objective function that:

(1) A $C_{\mathrm{p}}-\lambda$ curve with a flatter top, which means a larger average $C_{\mathrm{p}}$ for a TSR range around $\lambda_{\mathrm{opt}}$, can be obtained by the proposed multi-point method. This $C_{p}-\lambda$ curve can remedy the defect that the turbine cannot be maintained at the optimal TSR during the MPPT dynamic process due to turbulence and rotor inertia.

(2) Because the multiple design TSRs and weighting coefficients in the objective function are determined by $p\left(U_{\lambda_{j}}\right)$, the $C_{\mathrm{p}}$ at the TSR interval with large inflow wind energy can be significantly improved. Hence, the optimized $C_{\mathrm{p}}-\lambda$ curve can be coordinated with the distribution of $p\left(U_{\lambda_{j}}\right)$ (or $E_{\text {inflow }}^{U_{\lambda_{j}}}$ ).

\section{The Multi-Point Optimization Method Considering the Maximum Power Point Tracking Dynamic Process}

Compared with the inverse design method [3-7], it is more convenient to use the direct numerical optimization method $[11,20,27-30]$ to solve multi-point optimization problems. Therefore, this paper chooses the direct method to achieve multi-point optimization for VSWT blades. The geometric shape of the blade is parameterized by the Bezier curve. The Bladed software is used to calculate the $C_{\mathrm{p}}-\lambda$ curves of the optimized blades so as to evaluate the objective function. Finally, the genetic algorithm (GA) is used to solve the optimization model. 


\subsection{Baseline Wind Turbine and Simulation Parameters}

(1) Baseline wind turbine. The 1.5 MW WindPACT turbine [26] (mentioned in Section 2.2) is chosen as the baseline. The geometry of the baseline blade is described in Table 1. The geometrical description of the chord length and twist angle for the radial position is shown in Figure 3.

(2) MPPT control. The OT control strategy $[15,16]$ is used to realize MPPT.

(3) Turbulent wind. Parameters for the Bladed software to generate the three-dimensional turbulent wind are summarized in Table 2. These parameters are determined in compliance with the IEC61400-1 regulation [31].

(4) $\Delta \lambda$ and $r_{\text {tot }}$ are set to 0.5 and $90.0 \%$, respectively.

Table 1. Geometry of the National Renewable Energy Laboratory (NREL) 1.5 MW wind turbine blade.

\begin{tabular}{ccccc}
\hline Element & Position $(\mathbf{m})$ & Chord $(\mathbf{m})$ & Twist $\left(^{\circ}\right)$ & Airfoil \\
\hline 1 & 1.75 & 1.95 & 11.10 & \\
2 & 2.86 & 1.95 & 11.10 & cylinder \\
3 & 5.08 & 2.27 & 11.10 & \\
4 & 7.30 & 2.59 & 11.10 & \\
5 & 9.51 & 2.74 & 10.41 & s818 \\
6 & 11.73 & 2.58 & 8.38 & \\
7 & 13.95 & 2.41 & 6.35 & \\
8 & 16.16 & 2.25 & 4.33 & \\
9 & 18.38 & 2.08 & 2.85 & \\
10 & 20.60 & 1.92 & 2.22 & \\
11 & 22.81 & 1.75 & 1.58 & \\
12 & 25.03 & 1.59 & 0.95 & \\
13 & 27.25 & 1.43 & 0.53 & \\
14 & 29.46 & 1.28 & 0.38 & \\
15 & 31.68 & 1.13 & 0.23 & \\
16 & 33.90 & 0.98 & 0.08 & \\
17 & 35.00 & 0.50 & 0.00 & \\
\hline
\end{tabular}

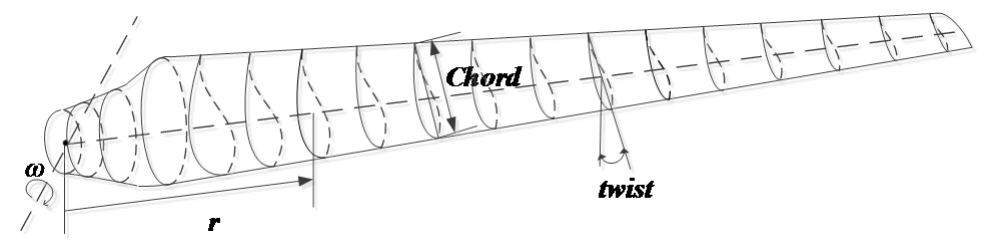

Figure 3. Geometrical description of the chord length and twist angle for the radial position.

Table 2. Parameters for generating 3D turbulent wind.

\begin{tabular}{ccc}
\hline Parameters & Value & Unit \\
\hline Mean wind speed & 5 & $\mathrm{~m} / \mathrm{s}$ \\
Height at which speed is defined & 84 & $\mathrm{~m}$ \\
Number of grid points in the lateral direction & 30 & - \\
Number of grid points in the vertical direction & 30 & - \\
Grid width & 150 & $\mathrm{~m}$ \\
Grid height & 150 & $\mathrm{~m}$ \\
Nyquist frequency of turbulent wind field & 27.7031 & $\mathrm{~Hz}$ \\
Time series length & 3600 & $\mathrm{~s}$ \\
Time step & 0.05 & $\mathrm{~s}$ \\
Turbulence model & IEC Kaimal & - \\
Turbulence class & $\mathrm{A}$ & - \\
\hline
\end{tabular}




\subsection{Determination of the Objective Function}

After the dynamic simulation of the closed-loop model for the baseline turbine is completed in Bladed, the proportion of the inflow wind energy corresponding to $U_{\lambda_{j}}$, i.e., $p\left(U_{\lambda_{j}}\right)$, can be calculated according to Equation (7). The results are shown in Figure 4.

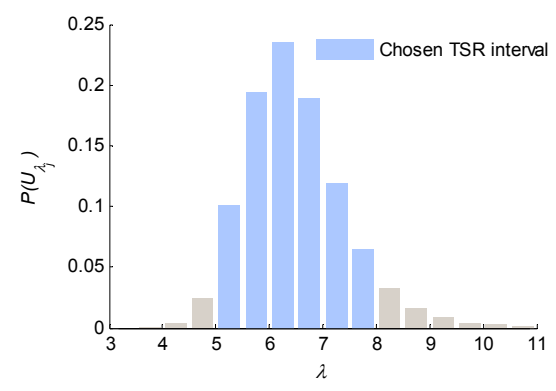

Figure 4. The proportion of the inflow wind energy corresponding to different tip speed ratio (TSR) intervals.

As shown in Figure 4, the $p\left(U_{\lambda_{j}}\right)$ corresponding to $U_{(5,8)}$ is $90.30 \%$. According to the selection method proposed in Section 4, the design TSR $\lambda_{j}$ and its corresponding weighting coefficients $\mu_{j}$ can be obtained, as listed in Table 3. Therefore, the objective function is constructed by taking these design TSRs and weighting coefficients to Equation (10).

Table 3. Design TSRs and weighting coefficients.

\begin{tabular}{ccc}
\hline $\boldsymbol{\lambda}_{j}$ & $\boldsymbol{p}\left(\boldsymbol{U}_{\boldsymbol{\lambda}_{j}}\right)$ & $\boldsymbol{\mu}_{j}$ \\
\hline 5.25 & $10.16 \%$ & 0.1125 \\
5.75 & $19.42 \%$ & 0.2150 \\
6.25 & $23.52 \%$ & 0.2604 \\
6.75 & $18.88 \%$ & 0.2091 \\
7.25 & $11.88 \%$ & 0.1315 \\
7.75 & $6.44 \%$ & 0.0714 \\
\hline
\end{tabular}

\subsection{Design Variables and Constraints}

The aerodynamic shape of a blade is defined by the chord length, twist angle and airfoil shape of each element in the radial position. Since airfoil optimization is beyond the scope of this study, only the chord and twist of each blade element are considered here. In order to achieve a continuous and smooth blade surface and reduce the number of design variables, two fourth order Bezier curves [11,32,33] are used to describe the radial distributions of the chord and twist of the elements $4-17$, as shown in Figures 5 and 6 .

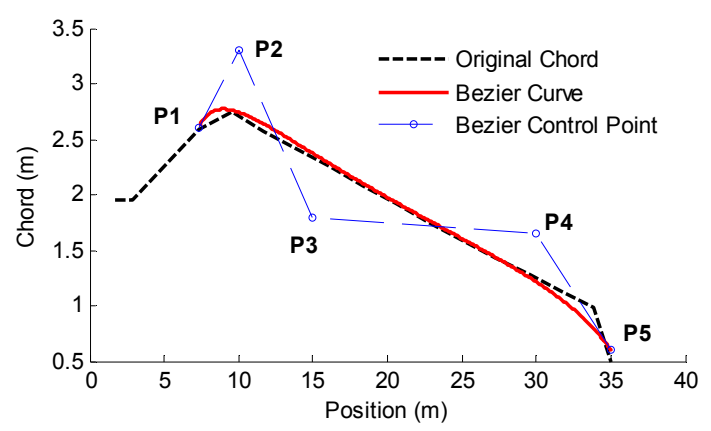

Figure 5. Bezier fitting curve and its control points of the original chord length. 


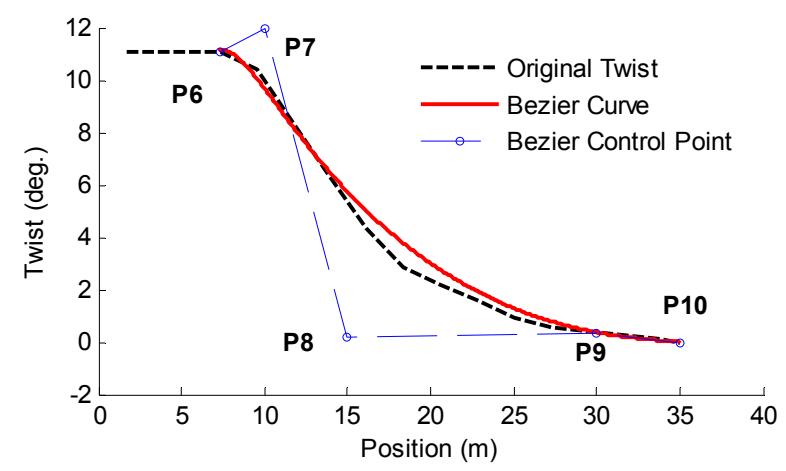

Figure 6. Bezier fitting curve and its control points for the original twist angle.

Because the part near the blade root closely connects to the rotor hub and does not produce much energy, the chord and twist of the elements 1-3 are fixed. In addition, the Bezier control points P1 and P6 are fixed to obtain a smoother transition between the elements 3 and 4 [11,30], and only the ordinates of the control points are allowed to alter freely. Therefore, only eight variables, regarded as the design variables, are needed to regenerate the chord and twist radial profiles.

The variation range of design parameters is listed in Table 4.

Table 4. Range of design variables.

\begin{tabular}{ccccccccc}
\hline Control Point & P2 & P3 & P4 & P5 & P7 & P8 & P9 & P10 \\
\hline original value & 3.3 & 1.8 & 1.7 & 0.6 & 12 & 0.2 & 0.3 & 0 \\
$\max$ & 5.0 & 4.0 & 3.0 & 1.0 & 14.0 & 1.0 & 0.8 & 0 \\
$\min$ & 2.0 & 1.5 & 1.0 & 0 & 9.0 & 0 & 0.5 & 0 \\
\hline
\end{tabular}

Furthermore, by analyzing the change in chord length in the existing blade optimizations $[11,20,34]$, the chord of each element of the optimized blade is set to no more than 1.05 times the original chord in order to save on material costs. Meanwhile, the effect of increased chord length on rotor inertia and the turbine's dynamic behavior can be limited and neglected. In addition, to keep the blade shape normal, the chord length and twist angle gradually decrease from the position of maximum value to the blade tip $[20,21,30]$.

\subsection{Optimization Scheme}

As a modern heuristic optimization algorithm, GA has the characteristics of parallel computing, high efficiency and global searching capability $[35,36]$. It has been widely used for aerodynamic optimization of wind turbine blades [27-30]. In this work, the solver "ga-Genetic Algorithm" in the MATLAB optimization toolbox is applied to search the global optimal solution that maximizes the objective function. Most parameters of the GA are set to default values except that the population size is set to 200. The optimization process is terminated if the number of iterations is more than 500 or the change in the best objective function value over 80 generations is less than $1 \times 10^{-8}$.

The flowchart of the blade optimization scheme is shown in Figure 7. Firstly, initialize the population which includes the original blade as an individual, and then import the variables of each individual to the Bezier curves to generate the corresponding blade shapes. For each blade shape chosen from the current population, check whether the geometry constraints are satisfied. If so, call Bladed software to obtain the $C_{\mathrm{p}}-\lambda$ curve of the blade and calculate the objective function; otherwise, set its objective function to zero. After all the individuals of the current population are evaluated, check whether the termination conditions of the GA are satisfied. If yes, terminate the optimization and output the optimized blade; otherwise, apply the GA operator to create the next population. 


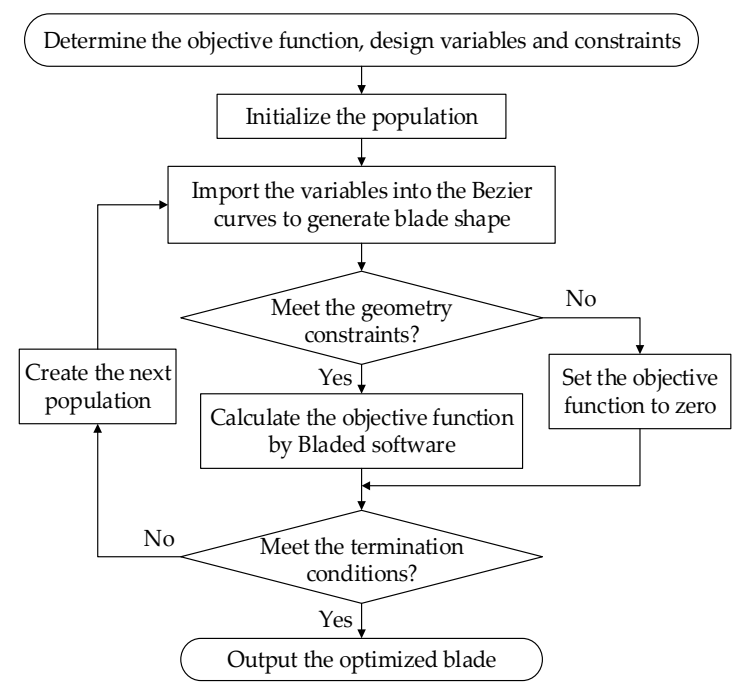

Figure 7. Flowchart of blade optimization scheme.

\section{Simulation Comparison and Analysis}

To verify the effectiveness of the multi-point optimization method, comparison and analysis are conducted between the multi-point and the conventional single-point methods in this section.

\subsection{Case Study of the Single-Point Design Method}

The single-point design method whose objective function is the maximization of $C_{\mathrm{p}}$ at a single design TSR is also used to optimize the original blade. It is worth noting that the conventional single-point design is generally based on the inverse method, which has a clear principle, analytical process and fast convergence. However, the smoothness of the surface of the optimized blade cannot be guaranteed. In addition, considering that the direct method is adopted in the multi-point design, it is also used to realize the single-point optimization design for higher comparability.

In this case, the objective function is to maximize the $C_{\mathrm{p}}$ at the optimal TSR of the baseline turbine, which is equal to 6.8 :

$$
o b j=\max \left\{C_{\mathrm{p}}\left(\lambda_{j}=6.8\right)\right\}
$$

The optimization process (including design variables and constraints) and simulation parameters are the same with the multi-point design case described in the Section 5.

\subsection{Aerodynamic Shape of the Optimized Blades}

The chord and twist angle distributions of the optimized and the original blades are shown in Figures 8 and 9 .

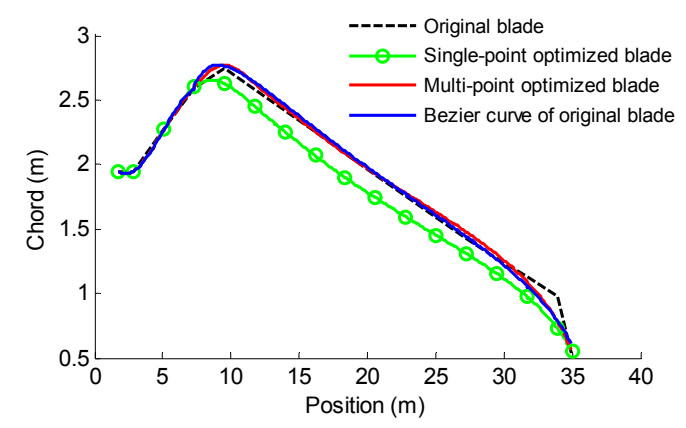

Figure 8. Chord distributions of the optimized and the original blades. 


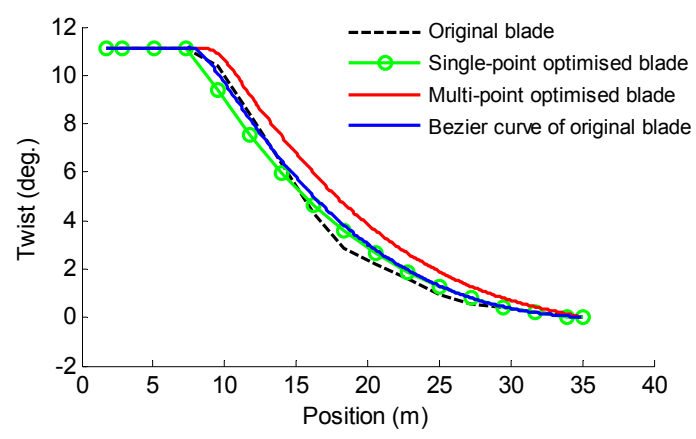

Figure 9. Twist angle distributions of the optimized and the original blades.

In Figure 8, the optimized blade obtained by the single-point design method (hereinafter referred to as "single-point blade") has a shorter chord compared with the original blade, while the chord of the optimized blade obtained by the multi-point design method (hereinafter referred to as "multi-point blade") maintains the original distribution at most part of the blade except at the region near the blade tip.

In Figure 9, the twist angle of the single-point blade is smaller than the original distribution at the inner elements (near the root) and it is bigger at the outer elements (near the tip), while the twist angle of the multi-point blade is bigger than the original distribution for most part of the blade. Based on the blade element theory [8,9], it can be inferred that a large twist angle puts the operational angle of attack of blade element far away from the stall angle of airfoil.

\subsection{Comparison of the Efficiency of Wind Energy Capture}

In order to verify the coordination between the static aerodynamic performance and the MPPT process, the fractional average power [37,38], as a closed-loop performance index of operating wind turbines under turbulent wind conditions, is used to evaluate the effectiveness of the aforementioned different blade design methods.

The fractional average power $P_{\text {favg }}$ is defined as the ratio of the mean captured power to the mean inflow wind power. This indicator reflects the mean efficiency of wind energy capture during the MPPT process for the VSWT operating in turbulent wind.

According to the simulated trajectories of wind turbines, the $P_{\text {favg }}$ is computed as:

$$
\begin{gathered}
P_{\text {favg }}=\frac{1}{N} \sum_{i=1}^{N} P_{\text {cap }}^{i} / \frac{1}{N} \sum_{i=1}^{N} P_{\text {inflow }}^{i} \\
P_{\text {cap }}^{i}=T_{\mathrm{e}, i} \omega_{\mathrm{e}, i}+J \omega_{i} \dot{\omega}_{i} \\
P_{\text {inflow }}^{i}=0.5 \rho \pi R^{2} v_{0, i}^{3}
\end{gathered}
$$

where $N$ is the total simulation steps, $P_{\text {cap }}^{i}, P_{\text {inflow' }}^{i} J \omega_{i} \dot{\omega}_{i}, T_{\mathrm{e}, i}$ and $\omega_{\mathrm{e}, i}$ are the captured wind power, inflow wind power, kinetic power, generator electromagnetic torque and generator speed at the $i$-th step, respectively.

For the same turbulent wind and MPPT control strategy (Note that the controller parameter $K_{\mathrm{opt}}$ needs to be modified by Equation (2) with the variation of $C_{\mathrm{p}, \max }$ and $\lambda_{\mathrm{opt}}$ ), the calculated $P_{\mathrm{favg}}$ and $C_{\mathrm{p}, \max }$ of different blades are listed in Table 5 . 
Table 5. Comparison of static aerodynamic performance and closed-loop performance.

\begin{tabular}{cccc}
\hline Blade & Original & Single-Point & Multi-Point \\
\hline$\lambda_{\text {opt }}$ & 6.8 & 7.0 & 6.9 \\
$C_{\mathrm{p}, \max }$ & 0.4727 & $0.4781(1.14 \%)$ & $0.4753(0.55 \%)$ \\
$P_{\text {favg }}$ & 0.4469 & $0.4483(0.30 \%)$ & $0.4527(1.29 \%)$ \\
\hline
\end{tabular}

Table 5 reveals that:

(1) The static aerodynamic performance at $\lambda_{\mathrm{opt}}$ (i.e., $C_{\mathrm{p}, \max }$ ) of the single-point blade is superior to that of the multi-point blade. Compared with the original blade, the $C_{\mathrm{p} \text {,max }}$ of the single-point blade is increased by $1.14 \%$, while the multi-point blade is increased by only $0.55 \%$, less than half of the single-point method.

(2) The closed-loop performance of the wind turbine applying the multi-point blade is better than that of the single-point blade. Compared with the original blade, the $P_{\text {favg }}$ corresponding to the multi-point and single-point blades is increased by $1.29 \%$ and $0.30 \%$, respectively.

In summary, an optimal static aerodynamic performance at a single point does not mean an optimal closed-loop performance of the operating wind turbine with multiple working points in turbulent wind. Moreover, since the coordination between static aerodynamic performance and MPPT dynamic process is considered in the determination of the objective function (Section 4), the multi-point method can further enhance the efficiency of wind energy capture under turbulent wind.

\subsection{Comparison between the Multi-Point and the Original Blades}

Figure 10 shows the $p\left(U_{\lambda_{j}}\right)$ and $C_{\mathrm{p}}-\lambda$ curves of the multi-point and the original blades. It can be observed that:

(1) The proportion of the inflow wind energy corresponding to each TSR interval, i.e., $p\left(U_{\lambda_{j}}\right)$, is similar between the multi-point and the original blades. This ensures that it is reasonable to determine the objective function based on the distribution of the inflow wind energy of the original blade.

(2) When the TSR is larger than 6.5 , the $C_{p}-\lambda$ curve of the multi-point blade is higher than the original one. While the TSR is smaller than 6.5 , the two curves are very close. Obviously, larger $C_{\mathrm{p}}$ in the interval $U_{(6.5,8)}$ results in a larger $P_{\text {favg }}$ of the turbine applying the multi-point blade.

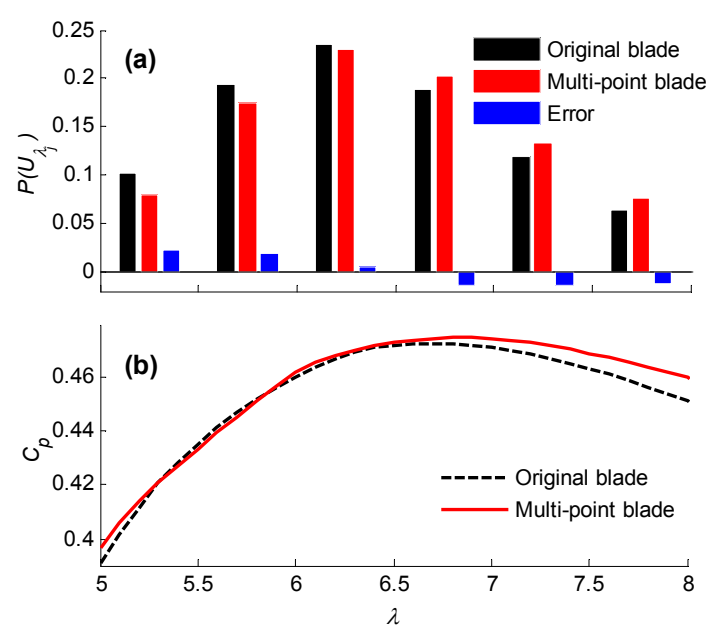

Figure 10. The proportion of the inflow wind energy: (a) the power coefficient; and (b) for the multi-point and the original blades. 
To further interpret the reason for the efficiency improvement of the multi-point optimized blade, the probability distributions of angles of attack (denoted by $P(\alpha)$ ) for two blade elements, which are respectively chosen from the original and multi-point optimized blades at $31.68 \mathrm{~m}$ away from the hub center and airfoil S826 [26], are compared in Figure 11.

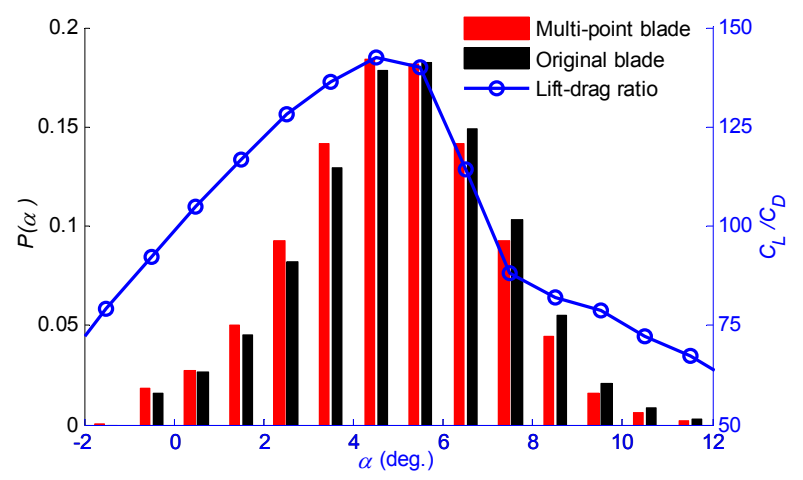

Figure 11. Probability distributions of angles of attack $\alpha$ for the blade elements chosen from the original and multi-point optimized blades.

As shown in Figure 11, compared with the original blade element, the probability of small angles of attack $\alpha\left(-2^{\circ}-5^{\circ}\right)$ for the multi-point blade element increases and meanwhile the probability of large $\alpha\left(5^{\circ}-12^{\circ}\right)$ decreases. This implies that the multi-point blade element spends more time operating at the $\alpha$ range where the lift-drag ratios $C_{\mathrm{L}} / C_{\mathrm{D}}$ are larger. This is the primary cause for the performance improvement of the multi-point blade.

\subsection{Comparison between the Multi-Point and the Single-Point Blades}

Figure 12 shows the $p\left(U_{\lambda_{j}}\right)$ and $C_{\mathrm{p}}-\lambda$ curves of the multi-point and the single-point blades. It can be seen from Figure 12 that:

(1) The top of $C_{p}-\lambda$ curve of the multi-point blade is flatter than that of the single-point one. Though the $C_{\mathrm{p}}$ of the multi-point blade is slightly smaller than the one of the single-point blade when the TSR is greater than 6.5, the former is significantly larger than the latter when the TSR is less than 6.5. For comparison, the distribution range of $\lambda_{\text {ope }}$ is divided into two TSR intervals: $U_{(5,6.5)}$ and $U_{(6.5,8)}$.

(2) Compared with the single-point blade, in the interval $U_{(5,6.5)}$ where the distribution of the inflow wind energy is more concentrated, the $C_{p}$ of the multi-point blade is significantly increased. Meanwhile, in the interval $U_{(6.5,8)}$ where the proportion of the inflow wind energy is smaller, the $C_{\mathrm{p}}$ of the multi-point blade is slightly decreased. This reflects the design idea described in Section 3 (namely focusing on improving the $C_{p}$ at the TSR interval where the inflow wind energy is primarily distributed) so that the static aerodynamic performance (i.e., $C_{\mathrm{p}}-\lambda$ curves) can be coordinated with the MPPT dynamic process (i.e., $p\left(U_{\lambda_{j}}\right)$ ).

(3) Furthermore, the captured wind energy corresponding to the interval $U_{(5,6.5)}$ and $U_{(6.5,8)}$ for the two blades are compared in Figure 13. It is shown that, although the wind energy captured by the turbine applying the multi-point blade is $8.5 \mathrm{MJ}$ less than that of the single-point blade in the interval $U_{(6.5,8)}$, the former is $14.6 \mathrm{MJ}$ more than the latter in the interval $U_{(5,6.5)}$. This results in a higher total captured wind energy for the turbine applying the multi-point blade. 

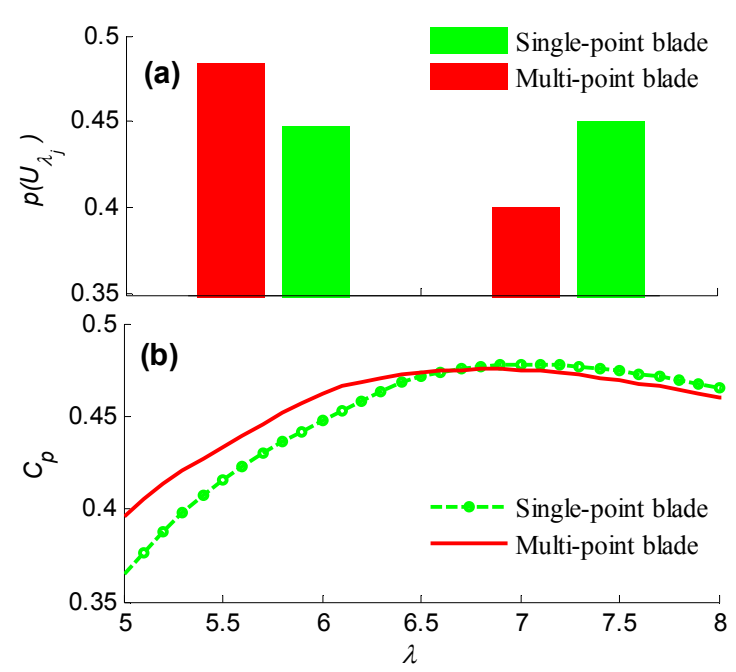

Figure 12. The proportion of the inflow wind energy: (a) the power coefficient; and (b) for the single-point and multi-point blades.

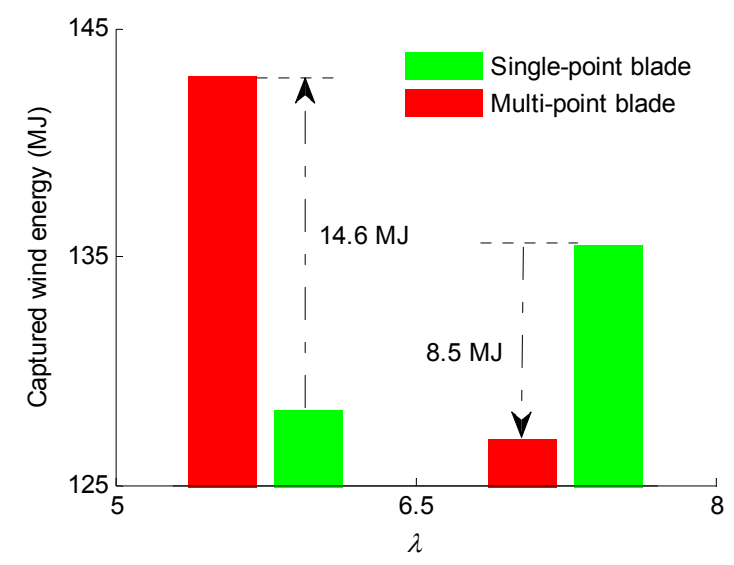

Figure 13. The captured wind energy corresponding to the interval $U_{(5,6.5)}$ and $U_{(6.5,8)}$.

\section{Conclusions}

The VSWTs cannot maintain the optimal TSR because of the MPPT dynamic process under turbulence. This implies that the conventional single-point design method, which only optimizes the power coefficient at a single TSR, needs to be improved. Also, the coupling and coordination between static aerodynamic performance and MPPT control should be considered in the aerodynamic design of VSWT blades to further increase the wind energy production of VSWTs.

In this paper, a multi-point optimization method considering the MPPT dynamic process was developed for aerodynamic design of VSWT blades. In this method, the inflow wind energy corresponding to each interval of the operational TSR for the original blade is determined through the time domain dynamic simulation. Then, by focusing on improving the power coefficient at the TSR where the corresponding inflow wind energy is relatively large, multiple TSRs and weighting coefficients in the objective function are thereby obtained. This method is validated by an optimization of the blade of an NREL 1.5 MW turbine.

The multi-point method is not a one-sided pursuit of a higher power coefficient at a single TSR, but is rather concerned with the average performance of multiple operating points. Therefore, the performance deficiency of the MPPT control is considered in the aerodynamic design. In other words, 
the decline of captured wind energy caused by the MPPT dynamic process is alleviated by coordinating the static aerodynamic performance of VSWTs with the MPPT dynamic process.

Acknowledgments: This work is supported by the National Natural Science Foundation of China (Nos. 61203129, 61174038, and 51507080), the Fundamental Research Funds for the Central Universities (Nos. 30915011104, 30920130121010, and 30920140112005) and China Southern Power Grid Company through the Project WYKJ00000027.

Author Contributions: Minghui Yin and Yun Zou proposed the motivation and improvement idea of the optimization strategy; Zhiqiang Yang built the optimization model and performed the numerical experiments; Yan $\mathrm{Xu}$ and Zhao Yang Dong provided guidance and key suggestions; Zhiqiang Yang, Minghui Yin, Yan Xu, Zhengyang Zhang, Yun Zou and Zhao Yang Dong wrote the paper.

Conflicts of Interest: The authors declare no conflict of interest.

\section{References}

1. Gómez-Iradi, S.; Barakos, G.N. Computational fluid dynamics investigation of some wind turbine rotor design parameters. Proc. Inst. Mech. Eng. Part A J. Power Energy 2008, 222, 455-470. [CrossRef]

2. Schubel, P.J.; Crossley, R.J. Wind turbine blade design. Energies 2012, 5, 3425-3449. [CrossRef]

3. Glauert, H. Airplane Propellers; Springer: Berlin/Heidelberg, Germany, 1935; pp. 169-360.

4. Wilson, R.E.; Lissaman, P.B.; Walker, S.N. Aerodynamic Performance of Wind Turbines; Oregon State University: Corvallis, OR, USA, 1976.

5. Sedaghat, A.; Mirhosseini, M. Aerodynamic design of a $300 \mathrm{~kW}$ horizontal axis wind turbine for province of Semnan. Energy Convers. Manag. 2012, 63, 87-94. [CrossRef]

6. Kim, B.; Kim, W.; Bae, S.; Park, J.; Kim, M. Aerodynamic design and performance analysis of multi-MW class wind turbine blade. J. Mech. Sci. Technol. 2011, 25, 1995-2002. [CrossRef]

7. Johansen, J.; Madsen, H.A.; Gaunaa, M.; Bak, C.; Sørensen, N.N. Design of a wind turbine rotor for maximum aerodynamic efficiency. Wind Energy 2009, 12, 261-273. [CrossRef]

8. Burton, T.; Sharpe, D.; Jenkins, N.; Bossanyi, E. Wind Energy Handbook; John Wiley \& Sons: Hoboken, NJ, USA, 2001.

9. Hansen, M.O. Aerodynamics of Wind Turbines; Routledge: Abingdon, UK, 2015.

10. Bavanish, B.; Thyagarajan, K. Optimization of power coefficient on a horizontal axis wind turbine using bem theory. Renew. Sustain. Energy Rev. 2013, 26, 169-182. [CrossRef]

11. Fuglsang, P.; Madsen, H.A. Optimization method for wind turbine rotors. J. Wind Eng. Ind. Aerodyn. 1999, 80, 191-206. [CrossRef]

12. Liu, X.; Wang, L.; Tang, X. Optimized linearization of chord and twist angle profiles for fixed-pitch fixed-speed wind turbine blades. Renew. Energy 2013, 57, 111-119. [CrossRef]

13. Lanzafame, R.; Messina, M. Optimal wind turbine design to maximize energy production. Proc. Inst. Mech. Eng. Part A J. Power Energy 2009, 223, 93-101. [CrossRef]

14. Kim, Y.S.; Chung, I.Y.; Moon, S.I. An analysis of variable-speed wind turbine power-control methods with fluctuating wind speed. Energies 2013, 6, 3323-3338. [CrossRef]

15. Abdullah, M.A.; Yatim, A.H.M.; Tan, C.W.; Saidur, R. A review of maximum power point tracking algorithms for wind energy systems. Renew. Sustain. Energy Rev. 2012, 16, 3220-3227. [CrossRef]

16. Bossanyi, E.A. Wind turbine control for load reduction. Wind Energy 2003, 6, 229-244. [CrossRef]

17. Bossanyi, E.A. The design of closed loop controllers for wind turbines. Wind Energy 2000, 3, 149-163. [CrossRef]

18. Tang, C.; Soong, W.L.; Freere, P.; Pathmanathan, M.; Ertugrul, N. Dynamic wind turbine output power reduction under varying wind speed conditions due to inertia. Wind Energy 2013, 16, 561-573. [CrossRef]

19. Morren, J.; Pierik, J.; De Haan, S.W. Inertial response of variable speed wind turbines. Electr. Power Syst. Res. 2006, 76, 980-987. [CrossRef]

20. Wang, X.; Shen, W.Z.; Zhu, W.J.; Sørensen, J.N.; Jin, C. Shape optimization of wind turbine blades. Wind Energy 2009, 12, 781-803.

21. Wang, T.; Wang, L.; Zhong, W.; Xu, B.; Chen, L. Large-scale wind turbine blade design and aerodynamic analysis. Chin. Sci. Bull. 2012, 57, 466-472. [CrossRef] 
22. Ning, S.A.; Damiani, R.; Moriarty, P.J. Objectives and constraints for wind turbine optimization. J. Sol. Energy Eng. 2014, 136. [CrossRef]

23. Bottasso, C.L.; Campagnolo, F.; Croce, A. Multi-disciplinary constrained optimization of wind turbines. Multibody Syst. Dyn. 2012, 27, 21-53. [CrossRef]

24. Bossanyi, E.A. GH Bladed Theory Manual; GH \& Partners Ltd.: New York, NY, USA, 2003.

25. Kim, B.; Kim, W.; Lee, S.; Bae, S.; Lee, Y. Developement and verification of a performance based optimal design software for wind turbine blades. Renew. Energy 2013, 54, 166-172. [CrossRef]

26. Malcolm, D.J.; Hansen, A.C. WindPACT Turbine Rotor Design Study; National Renewable Energy Laboratory: Golden, CO, USA, 2002.

27. Pourrajabian, A.; Ebrahimi, R.; Mirzaei, M. Applying micro scales of horizontal axis wind turbines for operation in low wind speed regions. Energy Convers. Manag. 2014, 87, 119-127. [CrossRef]

28. Lee, S.; Lee, S.; Ryi, J.; Choi, J.S. Design optimization of wind turbine blades for reduction of airfoil self-noise. J. Mech. Sci. Technol. 2013, 27, 413-420. [CrossRef]

29. Méndez, J.; Greiner, D. Wind blade chord and twist angle optimization by using genetic algorithms. In Proceedings of the Fifth International Conference on Engineering Computational Technology, Las Palmas de Gran Canaria, Spain, 12-15 September 2006; Civil-Comp Press: Stirling, UK, 2006.

30. Jeong, J.; Park, K.; Jun, S.; Song, K.; Lee, D.H. Design optimization of a wind turbine blade to reduce the fluctuating unsteady aerodynamic load in turbulent wind. J. Mech. Sci. Technol. 2012, 26, 827-838. [CrossRef]

31. Wind Turbine_Part 1: Design Requirements; IEC 61400-1; International Electrotechnical Commission: Geneva, Switzerland, 2005.

32. Hao, X.; Zhang, W.; Liu, X.; Liu, J. Aerodynamic and aeroacoustic optimization of wind turbine blade by a genetic algorithm. In Proceedings of the 46th AIAA Aerospace Sciences Meeting and Exhibit, Reno, NV, USA, 7-10 January 2008.

33. Benini, E.; Toffolo, A. Optimal design of horizontal-axis wind turbines using blade-element theory and evolutionary computation. J. Sol. Energy Eng. 2002, 124, 357-363. [CrossRef]

34. Fingersh, L.J.; Hand, M.M.; Laxson, A.S. Wind Turbine Design Cost and Scaling Model; National Renewable Energy Laboratory: Golden, CO, USA, 2006.

35. Mitchell, M. An Introduction to Genetic Algorithms; MIT Press: Cambridge, MA, USA, 1998.

36. Winter, G.; Periaux, J.; Galan, M.; Cuesta, P. Genetic Algorithms in Engineering and Computer Science; John Wiley \& Sons, Inc.: Hoboken, NJ, USA, 1996.

37. Johnson, K.E.; Pao, L.Y.; Balas, M.J.; Fingersh, L.J. Control of variable-speed wind turbines: Standard and adaptive techniques for maximizing energy capture. IEEE Control Syst. 2006, 26, 70-81. [CrossRef]

38. Johnson, K.E.; Fingersh, L.J.; Balas, M.J.; Pao, L.Y. Methods for increasing region 2 power capture on a variable-speed wind turbine. J. Sol. Energy Eng. 2004, 126, 1092-1100. [CrossRef] 